\title{
Nuclear Localization Leucine-Rich-Repeat Protein 1 Deficiency Protects Against Cardiac Hypertrophy by Pressure Overload
}

\author{
Jing Zong ${ }^{a, b}$ Fang-fang Li $i^{a, b}$ Kai Liang ${ }^{a, b}$ Rui Dai ${ }^{a, b}$ Hao Zhang ${ }^{a, b}$ Ling Yan ${ }^{c}$ \\ Jia-li Liu ${ }^{a, b}$ Lu-hong Xua,b Wen-hao Qian ${ }^{a, b}$
}

aDepartment of Cardiology, The Affiliated Hospital of Xuzhou Medical University, Xuzhou, ' ${ }^{2}$ Institute of Cardiovascular Disease Research, Xuzhou Medical University, Xuzhou, 'Medical Technology Institute of Xuzhou Medical University, Xuzhou, China

\section{Key Words}

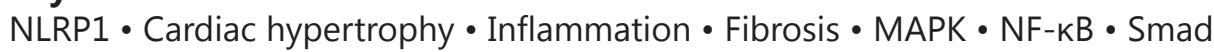

\begin{abstract}
Background/Aims: Nuclear localization leucine-rich-repeat protein 1 (NLRP1) is a cytoplasmic protein, involved in autoimmune diseases, mammalian reproduction, neuronal cell death, and stroke. However, the role of NLRP1 in cardiac hypertrophy remains unclear. We used in vivo and in vitro models to investigate the effects of NLRP1 on cardiac hypertrophy. Methods: We used NLRP1-deficient mice and cultured neonatal rat cardiomyocytes with gain and loss of NLRP1 function. Cardiac hypertrophy was estimated by echocardiographic and hemodynamic measurements, and by pathological and molecular analysis. Results: Eight weeks after aortic banding (AB), NLRP1 deficiency significantly inhibited aortic banding-induced cardiac hypertrophy, inflammation, and fibrosis. Activation of MAPK, NF-KB, and TGF- $\beta / \mathrm{Smad}$ pathways was reduced in NLRP1-knockout (KO) mice compared with that in wild-type (WT) mice. Consistent with these results, in vitro studies, performed using cultured neonatal mouse cardiomyocytes, confirmed that NLRP1 deficiency protects against cardiomyocyte hypertrophy induced by isoproterenol (PE); this protective activity was associated with the arrest of MAPK and NF-KB signaling. Conclusions: Our data illustrates that NLRP1 plays a crucial role in the development of cardiac hypertrophy via positive regulation of the MAPK, NF-KB, and TGF- $\beta$ / Smad signaling pathways.

\section{Introduction}

Cardiovascular disease is a major cause of death worldwide. Approximately 17 million people die of cardiovascular disease each year [1]. In China, the incidence of cardiovascular disease has increased gradually. Heart failure, the world's fastest-growing cardiovascular J. Zong and F.-f. Li contributed equally to this work.


condition, is the terminal stage of numerous cardiovascular diseases, such as hypertension, myocardial infarction, valvular disease, and congenital heart disease [2]. Hypertrophy of the heart is a common pathological process in these diseases [3]. Cardiac hypertrophy is initially a compensatory response to enhance myocardial contraction. The existence of continuous stimulation causes this compensation to gradually turn into decompensation, leading to alterations in the gene expression profile, cardiac systolic dysfunction, and extracellular remodeling [4,5]. Cardiac hypertrophy has become one of the independent risk factors for cardiovascular disease [6]. However, the pathogenesis of cardiac hypertrophy remains unclear, and there are no effective prevention or treatment measures. Therefore, it is important to find new targets for the prevention and treatment of cardiac hypertrophy.

Recent studies have shown that the inflammatory response and cell-signal transduction pathways are closely related to cardiac hypertrophy and heart failure $[7,8]$. The inflammatory reactions, which include the markers and prognostic indicators of cardiac hypertrophy and heart failure, are also involved in the pathogenesis of cardiac hypertrophy, induction of cardiomyocyte hypertrophy and embryonic gene expression, myocardial interstitial fibrosis, and cardiomyocyte apoptosis $[9,10]$. The nuclear localization leucine-rich-repeat protein 1 (NLRP1, also known as NALP1, DEFCAP, NAC, and CARD7) is a cytoplasmic protein that acts by forming a complex with the inflammasome; this promotes caspase-1- dependent processing of bioactive interleukin $-1 \beta$ (IL-1 $\beta$ ), resulting in the secretion of IL- $1 \beta$ and activation of the downstream inflammatory cascade [11]. NLRP1 is associated with autoimmune diseases, such as vitiligo, Addison's disease, type 1 diabetes, and rheumatoid arthritis [12]. The NLRP1 gene is involved in mammalian reproduction [13]. Overexpression of NLRP1 can lead to cellular apoptosis $[11,14]$. NLRP1 also plays an important role in neuronal cell death and stroke-induced behavioral deficits in mice [15]. However, there are no reports on the role of NLRP1 in cardiac hypertrophy. The NLRP subfamily (also known as the NALP family) belongs to the NLR family characterized by the pyrin domain. NLRP1 contains a pyrin domain which can bind to ASC, a nucleotide-binding domain (also called NATCH), a discovery domain, a CARD domain, and a leucine rich repeat domain [16].

Previous studies have shown that the occurrence and development of cardiac hypertrophy is the result of multiple signaling pathways. Signal transduction pathways involving mitogen-activated protein kinases (MAPKs), phosphatidylinositol-3 kinase (PI3K) /Akt, nuclear factor kappa B (NF-kB), and transforming growth factor beta 1 (TGF- $\beta 1$ ) / Smad play important roles in the development of cardiac hypertrophy.

However, the effect of NLRP1 on cardiac hypertrophy, especially when induced by pathological stimuli, has not been determined. The purpose of this study was to investigate the effects of NLRP1 on cardiac function and to elucidate the molecular mechanisms of NLRP1 in cardiac hypertrophy.

\section{Materials and Methods}

\section{Animals and animal models}

All animal procedures were performed in accordance with the Guide for the Care and Use of Laboratory Animals of the Chinese Animal Welfare Committee and the guidelines of our hospital. Adult male (8-10 weeks old) C57/BL6 and NLRP1 knockout mice were used in this study. NLRP1 knockout mice (B6.129S6Nlrp1btm1Bhk/J) were ordered from Jackson Laboratory (stock number 021301). The diet was based on commonly used diets in rodent intervention studies. Aortic banding (AB) was performed as described previously [17]. Surgery and subsequent analyses were performed in a blinded manner for all the groups. Mice were randomly assigned into four groups: $\mathrm{WT}+\mathrm{Sham}, \mathrm{WT}+\mathrm{AB}, \mathrm{KO}+\mathrm{Sham}$, and $\mathrm{KO}+\mathrm{AB}$. After the mice were euthanized, the hearts were dissected and weighed. We compared the ratios of heart weight/body weight (HW/BW, mg/g), lung weight/body weight (LW/BW, mg/g), and heart weight/tibia length (HW/TL, $\mathrm{mg} / \mathrm{mm}$ ) in WT and KO mice. 


\section{Cellular Physiology Cell Physiol Biochem 2018;48:75-86 \\ \begin{tabular}{l|l} 
and Biochemistry Published 10.1159/000491664 2018 & $\begin{array}{l}\text { () 2018 The Author(s). Published by S. Karger AG, Basel } \\
\text { www.karger.com/cpb }\end{array}$
\end{tabular}}

Zong et al.: Nuclear Localization Leucine-Rich-Repeat Protein 1 Promotes Cardiac Hypertrophy

\section{Echocardiography and hemodynamics}

Echocardiography was performed by Mylab30CV (ESAOTE S.P.A) with a $10 \mathrm{MHz}$ linear array ultrasound transducer. The left ventricle (LV) was assessed in both parasternal long-axis and short-axis views at a frame rate of $50 \mathrm{~Hz}$. End-systole and end-diastole were defined as the phase in which the smallest or largest area of LV was obtained, respectively. The left ventricular end-diastolic diameter (LVEDD) and left ventricular end-systolic diameter (LVESD) were measured using the LV M-mode tracing with a sweep speed of $50 \mathrm{~mm} / \mathrm{s}$ at the mid-papillary muscle level.

For hemodynamic measurements, mice were anesthetized with $1.5 \%$ isoflurane. A microtip catheter transducer (SPR-839, Millar Instruments, Houston, TX, USA) was inserted into the right carotid artery and advanced into the left ventricle. The pressure signals and heart rate were recorded continuously with a Millar Pressure-Volume System (MPVS-400, Millar Instruments, Houston, TX, USA), and the data were processed by PVAN data analysis software.

\section{Histological analysis}

Hearts were excised, washed with saline solution, and placed in $10 \%$ formalin. Hearts were cut transversely close to the apex to visualize the left and right ventricles. Several sections of the heart (4-5 $\mu \mathrm{m}$ thick) were prepared and stained with hematoxylin and eosin (H\&E) for histopathology, or with picrosirius red (PSR) for collagen deposition, and visualized by light microscopy. To examine the myocyte cross-sectional area, membranes in the sections were stained with FITC-conjugated wheat germ agglutinin (WGA; Invitrogen) and nuclei were stained with 4',6-diamidino-2-phenylindole (DAPI). A single myocyte was measured with a digital quantitative image digital analysis system (Image Pro-Plus, version 6.0). The outline of 100 myocytes was traced in each group.

\section{Cultured neonatal rat cardiac myocytes and treatment with recombinant adenoviral vectors}

Neonatal rat cardiomyocytes (NRCMs) were prepared as previously described [18]. Briefly, 1 to 2-dayold Sprague-Dawley rats were euthanized and the neonatal hearts were removed from the thoracic cavities. The heart tissues were finely minced and digested in phosphate buffered saline (PBS) containing $0.03 \%$ trypsin and $0.04 \%$ collagenase type II. After removing fibroblasts using a differential attachment technique, NRCMs were seeded at a density of $1 \times 10^{6}$ cells/well onto gelatin-coated six-well culture plates. A medium, consisting of DMEM/F12 supplemented with $20 \%$ fetal calf serum (FCS), BrdU (0.1 mM, used to inhibit the proliferation of fibroblasts), and penicillin/streptomycin was then added to these six-well plates. After 48 hours, the culture medium was replaced with serum-free DMEM/F12 for 12 hours prior to stimulation with phenylephrine (PE, $10 \mu \mathrm{M})$.

Replication-defective adenoviral vectors were used to overexpress rat NLRP1 (Ad- NLRP1) under the control of a cytomegalovirus promoter. A similar adenoviral vector, expressing green fluorescent protein (Ad-GFP), was used as a control. The rat Ad-shNLRP1 was used to knock down the expression of NLRP1, and Ad-shRNA was used as the nontargeting control. The neonatal rat cardiomyocytes were infected for 8 hours with Ad-GFP, Ad-NLRP1, Ad-shRNA, and Ad-shNLRP1 in diluted media at a multiplicity of infection (MOI) of 100.

To identify the role of NLRP1 in cardiac hypertrophy, we treated Ad-shRNA-infected, Ad-shNLRP1infected, Ad-GFP-infected, and Ad-NLRP1-infected cardiomyocytes with isoproterenol (PE, $10 \mu \mathrm{M})$ for 24 hours.

\section{Immunofluorescence}

NRCMs were infected with different adenoviruses for 24 hours and then permeabilized with $0.1 \%$ Triton X-100 in PBS for 50 minutes. To determine the cell-surface areas, NRCMs were then labeled with $\alpha$-actinin (Abcam, ab68194, 1:200 dilution) and cardiac troponin T (Abcam, ab8295, 1:100 dilution). NRCMs were then reacted with a FITC-labeled goat anti-rabbit secondary antibody (Aspen, AS-1110, 1:50 dilution) and a Cy3-labeled goat anti-mouse secondary antibody (Aspen, AS-1111, 1:50 dilution).

\section{Quantitative real-time PCR.}

Real-time PCR was used to detect RNA expression levels of hypertrophic and fibrotic markers. Total RNA was extracted from frozen, pulverized mouse cardiac tissue using TRIzol (Roche). cDNA was synthesized using oligo(dT) primers with an Advantage RT-for-PCR kit (Roche). We performed PCR with LightCycler 480 


\section{Cellular Physiology Cell Physiol Biochem 2018;48:75-86 \begin{tabular}{l|l} 
DOI: 10.1159/000491664 & $\begin{array}{l}\text { O } 2018 \text { The Author(s). Published by S. Karger AG, Basel } \\
\text { www.karger.com/cpb }\end{array}$
\end{tabular}}

Zong et al.: Nuclear Localization Leucine-Rich-Repeat Protein 1 Promotes Cardiac Hypertrophy

SYBR Green 1 Master Mix (Roche), and normalized the results against the expression of glyceraldehyde-3phosphate dehydrogenase (GAPDH).

\section{Western blotting}

Mouse ventricles and cultured neonatal rat cardiac myocytes from the different groups were lysed in RIPA lysis buffer. Fifty micrograms of cell lysate was used for SDS/PAGE, and proteins were subsequently transferred to an Immobilon-P membrane (Millipore, Beijing, China). The specific protein expression levels were normalized to that of GAPDH for total cell lysates and cytosolic proteins on the same polyvinylidene difluoride membrane. The primary antibodies were specific for: p-JNK1/2 (Cell Signaling Technology, 4668P), T-JNK1/2 (Cell Signaling Technology, 9258), p-p38 (Cell Signaling Technology, 4511P), T-p38 (Cell Signaling Technology, 9212P), p-ERK1/2 (Cell Signaling Technology, 4370P), T-ERK1/2 (Cell Signaling Technology, 4695), TGF- $\beta$ (Abcam, ab66043), TGF- $\beta$ Receptor I (Abcam, ab31013), TGF- $\beta$ Receptor II (Abcam, ab61213), p-Smad2 (Cell Signaling Technology, 3101), Smad2 (Cell Signaling Technology, 3103s), p-Smad3 (Cell Signaling Technology, 8769), Smad3 (Santa Cruz Biotechnology, sc-101154), Smad4 (Santa Cruz Biotechnology, sc-7966), and GAPDH (Cell Signaling Technology, 2118). The bicinchoninic acid protein assay kit was purchased from Pierce. The secondary antibodies, goat anti-rabbit IRdye@ 800 CW (LI-COR, 926-32211) IgG and goat anti-mouse IRdye@ 800 CW (LI-COR, C11026-03) IgG, were used at 1:10000 in Odyssey blocking buffer for 1 hour. The blots were scanned with an infrared Li-Cor scanner, allowing for the simultaneous detection of two targets (antiphospho and anti-total protein) in the same experiment.

Protein extracts $(40 \mu \mathrm{g})$ of the myocardia from different groups were fractionated on a $10 \%$ SDSPAGE (ASPEN, AS1012) and transferred to PVDF membranes (Millipore, IPVH00010). The membrane was blocked with $5 \%$ non-fat milk for 1 hour and incubated with the primary antibody at $4{ }^{\circ} \mathrm{C}$ overnight. The primary antibodies were specific for: ANP (Abcam, ab189921), BNP (Biorbyt, orb382908), $\beta$-MHC (Abcam, ab180779), NLRP1 (Cell Signaling Technology, 4990), p-IKK $\beta$ (Affbiotech, AF3010), IKK $\beta$ (Abcam,

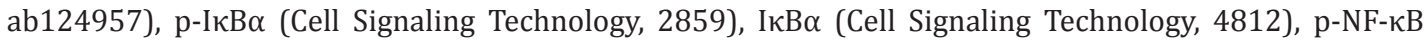
p65(Cell Signaling Technology, 3033), NF-кB p65(Cell Signaling Technology, 8242), and GAPDH (Abcam, ab37168). The membrane was then incubated with HRP-conjugated secondary antibody (ASPEN, AS1107) at 1:10000 dilutions for 1 hour at room temperature. After application of a chemiluminescence reagent, membranes were exposed to X-ray films.

\section{Statistical analysis}

Data are expressed as the means \pm SEM. Differences among groups were assessed by two-way ANOVA followed by a post hoc Tukey's test. Comparisons between two groups were performed by unpaired Student's $t$-test. $P<0.05$ was considered statistically significant.

\section{Results}

NLRP1 expression in mouse cardiac hypertrophy induced by pressure overload

To explore the potential role of NLRP1 in cardiac hypertrophy, we investigated the expression of NLRP1 in response to hypertrophic stresses in mice. The expression levels of the NLRP1 protein were elevated for 1 to 8 weeks after AB. The levels of hypertrophic makers, such as atrial natriuretic peptide (ANP), B-type natriuretic peptide (BNP), and $\beta$-myosin heavy chain ( $\beta$-MHC), were higher in WT mice for 1 to 8 weeks after AB (Fig. 1 ). This indicates that the expression and activity of NLRP1 were enhanced in response to hypertrophic stimuli, and that NLRP1 may participate in cardiac hypertrophy.

\section{NLRP1 deficiency alleviates cardiac hypertrophy and dysfunction induced by $A B$}

To determine how the absence of NLRP1 affects the hypertrophic response to pressure overload, we used NLRP1 KO mice and WT littermates (Fig. 2a); both groups of mice were subjected to $\mathrm{AB}$ or a sham procedure. After 8 weeks, we assessed the cardiac function of KO and WT mice by echocardiography and pressure-volume (PV) loop. The increases in the dimensions of the LV chamber and interventricular septal thickness, induced by pressure overload, were significantly attenuated during both the systole and diastole in KO mice 
Zong et al.: Nuclear Localization Leucine-Rich-Repeat Protein 1 Promotes Cardiac Hypertrophy

Fig. 1. NLRP1 expression in hypertrophic heart. Western blotting was used to examine the levels of the cardiac proteins atrial natriuretic peptide (ANP), brain natriuretic peptide (BNP), $\beta$-MHC, and NLRP1 in wild-type (WT) mice after aortic banding (AB) at the time points indicated. Top, representative blots; bottom, quantitative results. ${ }^{*} P<0.05$ vs sham group.

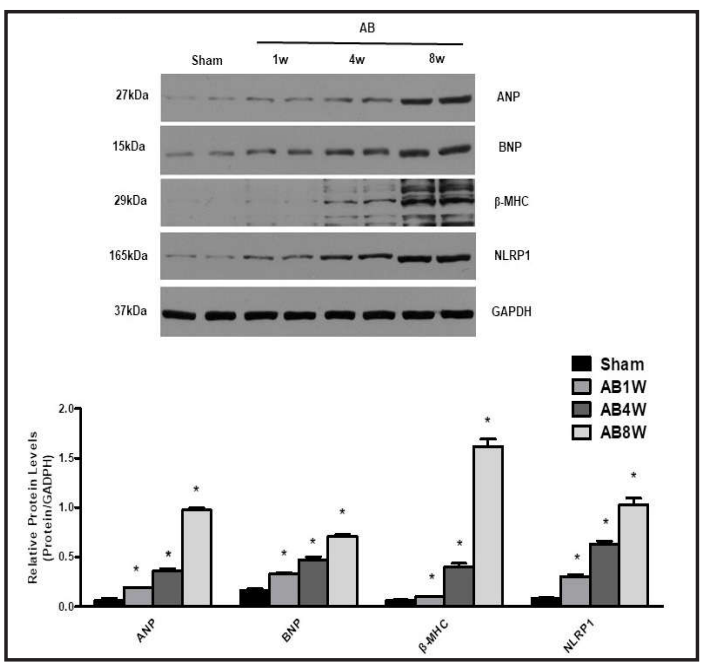

Fig. 2. Effects of NLRP1 deficiency on cardiac hypertrophy. (a) Deletion of NLRP1 was confirmed by western blotting $(n=4)$. WT represents wild-type hearts, and KO represents NLRP1-knockout hearts. (b) Statistical analysis of heart weight/body weight (HW/ BW), lung weight/body weight (LW/BW), and heart weight/tibia length $(\mathrm{HW} / \mathrm{TL})$ of sham and $\mathrm{AB}$ mice at 8 weeks post $A B$ surgery. (c) Gross hearts, hematoxylin and eosin (H\&E) staining, wheat germ agglutinin (WGA)-FITC staining, and myocyte cross-sectional areas of in indicated groups. (d) The expression of ANP, BNP, $\beta$-MHC, $\alpha-\mathrm{MHC}$, and SERCA2a, induced by $A B$, was determined by real-time PCR. Values are the mean \pm SEM. ${ }^{*} P<0.05$ vs the corresponding sham group. ${ }^{\#} P<0.05$ vs $\mathrm{WT}+\mathrm{AB}$ group.

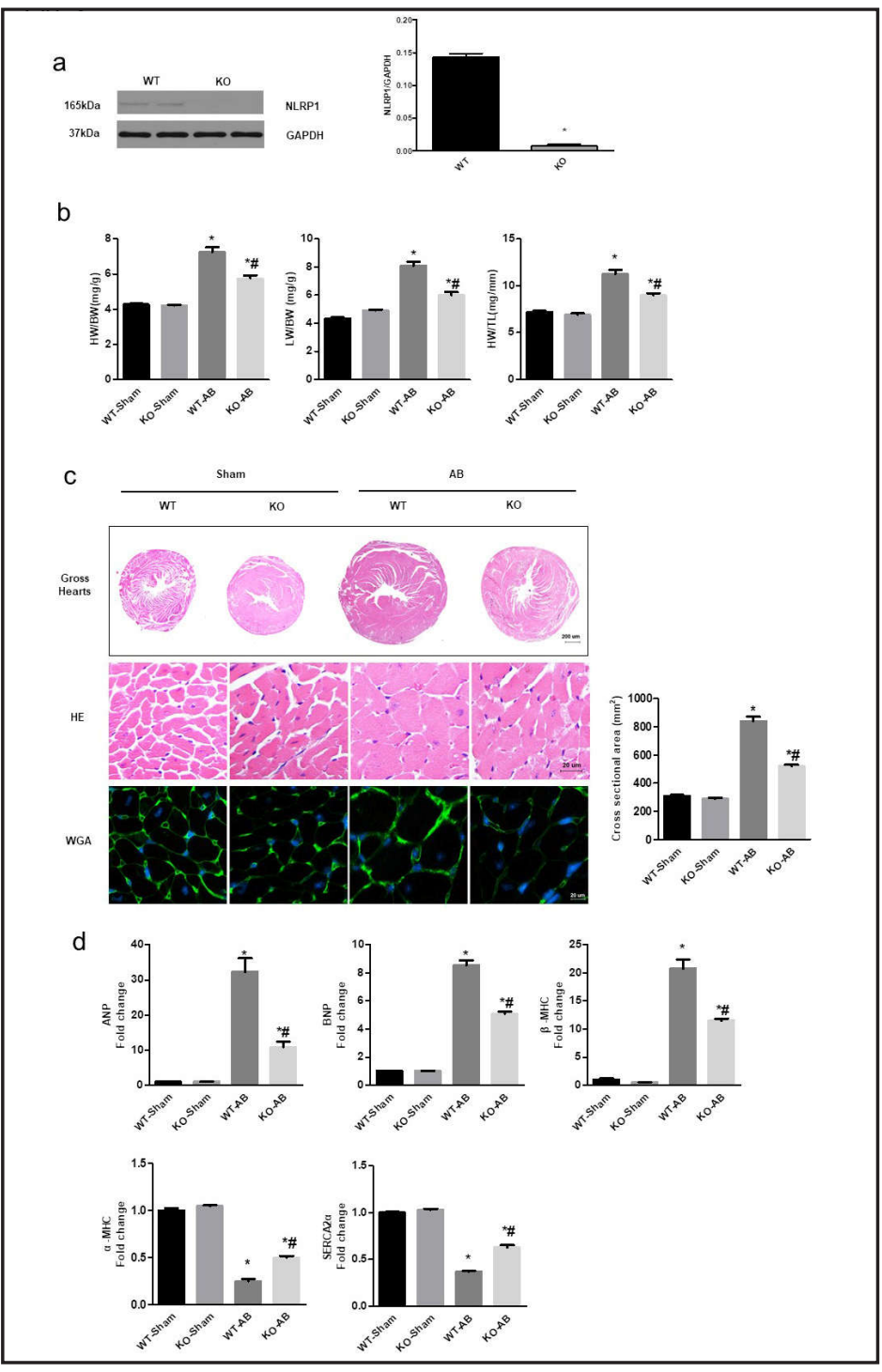




\section{Cellular Physiology Cell Physiol Biochem 2018;48:75-86

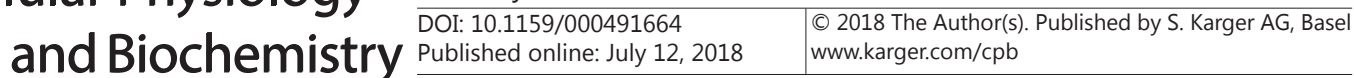

Zong et al.: Nuclear Localization Leucine-Rich-Repeat Protein 1 Promotes Cardiac Hypertrophy

compared with those in WT mice. LV contraction was significantly improved in the KO hearts, as determined by measuring both the LV ejection fraction (EF) and fractional shortening (FS) (Table 1). Using pressure-volume loop analysis, we further examined the aggravated hemodynamic dysfunction of the LV in the KO mice; for this, we measured parameters that reflect the LV volume, and systolic and diastolic function (Table 1). NLRP1 KO mice showed attenuated cardiac hypertrophy 8 weeks after $\mathrm{AB}$. These mice had decreased ratios of heart weight/body weight (HW/BW), lung weight/body weight (LW/BW), and heart weight/tibia length (HW/TL) (Fig. $2 \mathrm{~b}$ ), as assessed by the morphology of the gross heart, H\&E staining, WGA staining, and cardiomyocyte cross-sectional area (Fig. 2c). The levels of hypertrophic markers, including ANP, BNP, and $\beta$-MHC, were reduced in the $\mathrm{KO}$ mice after pressure overload. This was accompanied by the upregulation of $\alpha$-myosin heavy chain $(\alpha-\mathrm{MHC})$ and sarcoendoplasmic reticulum $\mathrm{Ca}^{2+}$-ATPase (SERCA2a) (Fig. 2d). These results indicate that ablation of NLRP1 abates cardiac hypertrophy and blocks impaired cardiac function after pressure overload.

\section{NLRP1 deficiency represses cardiomyocyte hypertrophy in vitro}

To further examine the effect of NLPR1 on cardiac hypertrophy, we treated cultured neonatal rat cardiomyocytes with PE $(10 \mu \mathrm{M})$. The cells were infected with Ad-GFP, AdNLRP1, Ad-shRNA, or Ad-shNLRP1 for 24 hours, then serum starved for 24 hours in $0.5 \%$ FCS. The cells then were exposed to either PE $(10 \mu \mathrm{M})$ or a PBS control for 24 hours (Fig. 3a). After stimulation with PE, the expression of NLRP1 was downregulated by infection with Ad-shNLRP1. This inhibited cell hypertrophy and mRNA expression of ANP, BNP, and $\beta$-MHC compared with those in the negative control (Ad-shRNA) group (Fig. 3b, d). Conversely, overexpression of NLRP1, induced via infection with Ad-NLRP1, promoted cell hypertrophy and mRNA expression of ANP, BNP, and $\beta$-MHC (Fig. 3c, e). These findings indicate that NLRP1 deficiency blocks cardiac hypertrophy in vitro.

\section{Effects of NLRP1 on MAPK signaling}

To explore the molecular mechanisms of NLRP1 in cardiac hypertrophy, we investigated the activation of mitogen-activated protein kinase (MAPK) pathway in KO and WT hearts in response to pressure overload. The levels of phosphorylated JNK1/2, p38, and ERK1/2 were significantly increased in the mice after $\mathrm{AB}$. However, this increased phosphorylation of JNK1/2, p38, and ERK1/2 was blocked in NLRP1-deficient hearts (Fig. 4a). Consistent with the results of our in vivo procedures, the activation of JNK1/2, p38, and ERK1/2 was markedly reduced in Ad-shNLRP1 cardiomyocytes compared with that of control cells after treatment with PE. In contrast, the levels of phosphorylated JNK1/2, p38, and ERK1/2 were upregulated in Ad-NLRP1 cardiomyocytes (Fig. 4b, c). These results indicate that MAPK signaling was vital for the effects of NLRP1 on cardiac hypertrophy. 
Fig. 3. NLRP1 regulates PE-induced cardiomyocyte hypertrophy in vitro. (a) The expression of NLRP1 was increased in Ad-NLRP1 cardiomyocytes (especially after stimulation with phenylephrine [PE]) and downregulated in AdshNLRP1 cardiomyocytes. Top: representative blots; bottom: quantitative results. (b and c) Representative images of cardiomyocytes infected with AdshRNA, Ad-shNLRP1, Ad-GFP, or Ad-NLRP1 for 8 hours and then incubated with $10 \mu \mathrm{M}$ PE for 24 hours. Left: representative images; right: quantitative results of cell surface area ( $\mathrm{n}=100+$ cells). (d and e) Real-time PCR analyses of the hypertrophic markers ANP, $\mathrm{BNP}$, and $\beta$-MHC in the indicated groups. Values are the mean \pm SEM. ${ }^{*} P<0.05$ vs the corresponding PBS group. ${ }^{\#} P<0.05$ vs Ad-shRNA/AdGFP in the PE group.

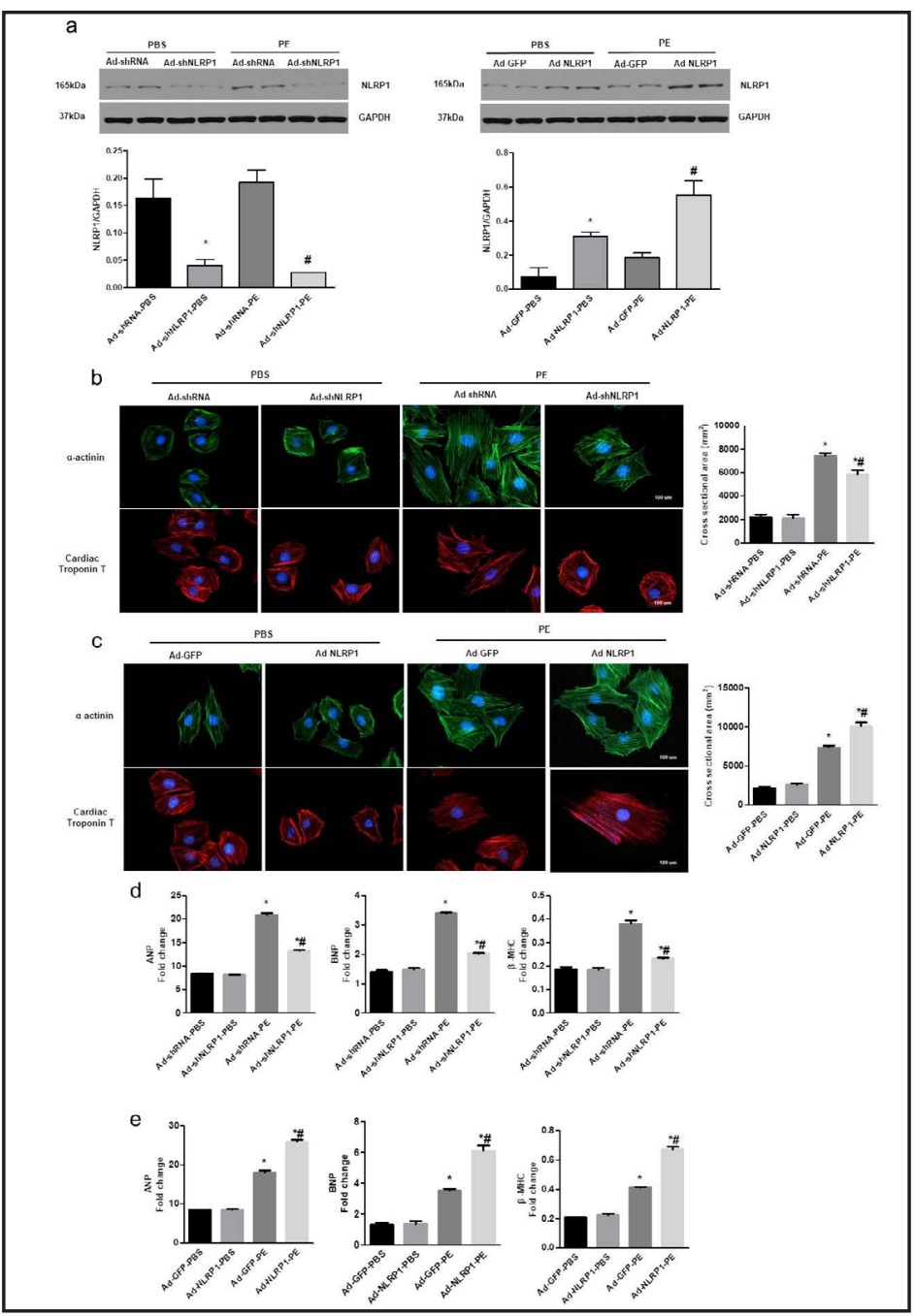

Fig. 4. Effects of NLRP1 on MAPK signaling. (a) The levels of total and phosphorylated JNK1/2, p38, and ERK1/2 in the heart tissues of mice in the indicated groups $(n=6)$. Top, representative blots; bottom, quantitative results. (b and c) The levels of total and phosphorylated JNK1/2, p38, and ERK1/2 in
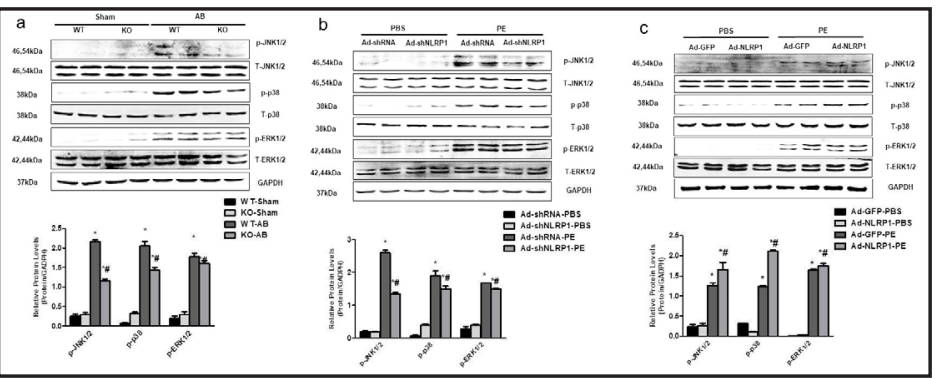
cardiomyocytes infected with AdshRNA, Ad-shNLRP1, Ad-GFP, or Ad-NLRP1 for 8 hours and then incubated with $10 \mu \mathrm{M}$ PE for 24 hours. Top, representative blots; bottom, quantitative results. Values are the mean \pm SEM. ${ }^{*} P<0.05$ vs the corresponding PBS group. ${ }^{*}<0.05$ vs Ad-shRNA/Ad-GFP in the PE group.

\section{NLRP1 ablation weakens cardiac inflammation}

Activation of inflammatory cytokines and NF- $\kappa \mathrm{B}$ signaling mediate the development and progression of cardiac hypertrophy [19]. We examined the mRNA expression of NF- $\kappa$ B target genes, including interleukin (IL)-1 $\beta$, IL-6, and tumor necrosis factor (TNF)- $\alpha$, in the cardiac tissue of $\mathrm{KO}$ mice and their WT littermates 8 weeks after AB (Fig. 5a). The phosphorylation of $\mathrm{IKK} \beta, \mathrm{IkB} \alpha$, and NF-kBp65 was significantly reduced, whereas the total level of $\mathrm{IkB} \alpha$ 
was higher post $\mathrm{AB}$ in $\mathrm{KO}$ mice compared with that in WT mice (Fig. 5b). Consistent with the results of our in vivo procedure, the mRNA expressions of IL-1 $\beta$, IL-6, and TNF- $\alpha$ was reduced in Ad-shNLRP1 cardiomyocytes compared with that of control cells after treatment with PE. In contrast, the mRNA expressions of IL-1 $\beta$, IL-6, and TNF- $\alpha$ was upregulated in Ad-NLRP1 cardiomyocytes (Fig. 5c). Compared with the levels observed in control cells, the phosphorylation of IKK $\beta$, IkB $\alpha$, and NF-kBp65 was significantly inhibited, whereas the total level of IkB $\alpha$ was higher, in Ad-shNLRP1 cardiomyocytes after treatment with PE. The

Fig. 5. Effect of NLRP1 on NF-kB signaling in vivo and in vitro. (a) The mRNA expressions of IL-1 $\beta$, IL10 , and TNF- $\alpha$ in the myocardium were assessed in the indicated groups using real-time PCR $(n=6)$. (b) Representative western blot analysis of phosphorylated and total inhibitor of $\kappa \mathrm{B}$ kinase- $\beta$ (IKK $\beta)$, inhibitor of NF- $\mathrm{B} \alpha(\mathrm{I} \kappa \mathrm{B} \alpha)$, and NF- $\kappa$ Bp65 in the heart tissues of mice from the indicated groups $(n=6)$. Top, representative blots;

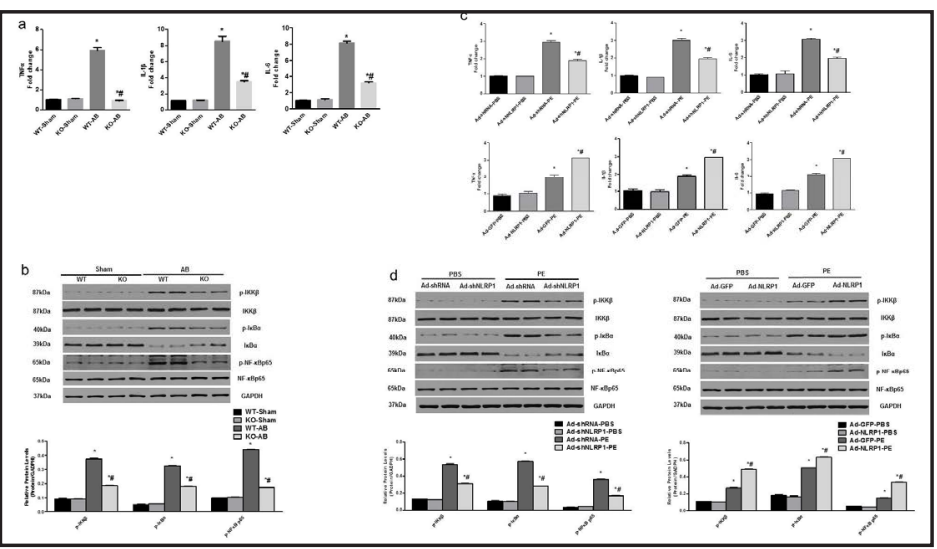
bottom, quantitative results. Values are the mean \pm SEM. ${ }^{*} P<0.05$ compared with the corresponding sham group. ${ }^{\#} P<0.05$ vs WT $+\mathrm{AB}$ group. (c) mRNA levels of IL-1 $\beta$, IL-10, and TNF- $\alpha$ in neonatal rat cardiomyocytes (NRCMs) infected with Ad-shRNA, Ad-shNLRP1, Ad-GFP, or Ad-NLRP1, and treated with PBS or $10 \mu \mathrm{M}$ PE for 24 hours. Top, representative blots; bottom, quantitative results. (d) Representative western blot analysis of phosphorylated and total IKK $\beta$, $\mathrm{I} \kappa \mathrm{B} \alpha$, and NF- $\kappa \mathrm{Bp} 65$ expression in cardiomyocytes obtained from the indicated groups. Top, representative blots; bottom, quantitative results. Values are the mean \pm SEM. ${ }^{*} P<0.05$ vs the corresponding PBS group. ${ }^{\#} P<0.05$ vs Ad-shRNA/Ad-GFP in the PE group.

Fig. 6. NLRP1 attenuates fibrosis induced by pressure overload. (a) Picrosirius red (PSR) staining of the histological sections of the left ventricle (LV) was performed in each group 4 weeks after $\mathrm{AB}$ or sham surgery. (b) Fibrotic areas in the sections were quantified using an image analysis system. (c) mRNA expression levels of collagen I, collagen III, and CTGF in the myocardium were obtained for the indicated groups using RT-PCR. ( $d$ and e) Western blot analysis of TGF- $\beta$, TGF- $\beta$ R I, TGF$\beta R$ II, p-Smad2, Smad2, p-Smad3, Smad3, and Smad4 expression in mice from the indicated groups; d, representative blots; e, quantitative results. Values are the mean \pm SEM. ${ }^{*} P<0.05$ compared with the corresponding sham

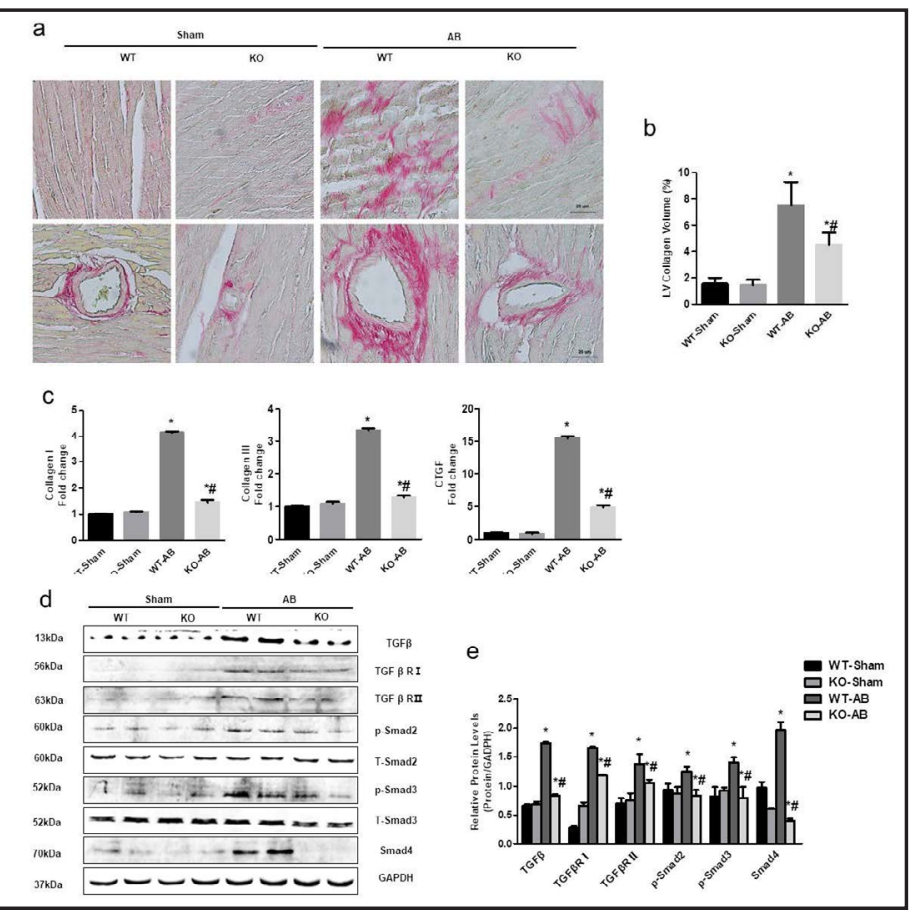
group. ${ }^{\#} P<0.05$ vs $\mathrm{WT}+\mathrm{AB}$ group. 


\section{Cellular Physiology Cell Physiol Biochem 2018;48:75-86 \\ \begin{tabular}{c|c} 
and Biochemistry Published online: July I2, 2018 & $\begin{array}{l}\text { O 2018 The Author(s). Published by S. Karger AG, Basel } \\
\text { www.karger.com/cpb }\end{array}$
\end{tabular}}

Zong et al.: Nuclear Localization Leucine-Rich-Repeat Protein 1 Promotes Cardiac Hypertrophy

phosphorylation of IKK $\beta$, IkB $\alpha$, and NF-kBp65 was significantly increased, whereas the total level of IkB $\alpha$ was lower, in Ad-NLRP1 cardiomyocytes after treatment with PE (Fig. 5d).

\section{NLRP1 ablation attenuates cardiac fibrosis induced by pressure overload}

Cardiac fibrosis is a characteristic feature of pathological cardiac hypertrophy. To further illustrate the mechanism by which NLRP1 ablation inhibits cardiac hypertrophy, heart sections were stained with PSR to detect left ventricular interstitial fibrosis. Perivascular and interstitial fibrosis, and LV collagen volume, were significantly increased in WT mice subjected to AB; however, cardiac fibrosis was markedly attenuated in KO mice compared with that in their WT littermates. Left ventricular interstitial fibrosis was evaluated by PSR conducted using paraffin-embedded sections. Perivascular and interstitial fibrosis was detected in both KO and WT mice, but the extent of cardiac fibrosis was markedly inhibited in KO mice (Fig. 6a, b). Moreover, the mRNA expression levels of known mediators of fibrosis, including collagen I, collagen III, and connective tissue growth factor (CTGF), were remarkably downregulated in KO mice compared with the levels in WT mice after treatment with AB (Fig. 6c). To further investigate whether NLRP1 deficiency can inhibit fibrosis, we assessed the regulatory role of NLRP1 in the activation of the TGF- $\beta / S m a d$ cascade. The protein-expression levels of TGF- $\beta$, TGF- $\beta$ R I, TGF- $\beta$ R II, p-Smad2, p-Smad3, and Smad4 were decreased in $\mathrm{KO}$ mice after $\mathrm{AB}$ (Fig. $6 \mathrm{~d}$, e). Collectively, these data suggest that NLRP1 deficiency plays an anti-fibrotic role by blunting the TGF- $\beta /$ Smad signaling.

\section{Discussion}

In this study, we used NLRP1 KO mice and neonatal rat cardiomyocytes infected with AdGFP, Ad-NLRP1, Ad-shRNA, and Ad-shNLRP1 to explore the role of NLRP1 in the hypertrophic response. Our results demonstrate that the ablation of NLRP1 protected against cardiac hypertrophy, inflammation, and fibrosis under conditions of pressure overload. The effects of NLRP1 on cardiac hypertrophy, inflammation, and fibrosis likely occur via regulation of the MAPK, NF- $\kappa$ B, and TGF- $\beta /$ Smad signaling pathways. These results suggest that NLRP1 may play an aggravating role in pathological hypertrophy of the heart.

The NLR protein family is divided into several sub-families according to differences in the $\mathrm{N}$-terminal domain [20]. Unlike humans, which have a single NLRP1 gene, the mouse NLRP1 gene has three homologues: Nlrp1a, Nlrp1b, and Nlrp1c [21]. NLRP1 mRNA is expressed in many tissues such as the heart, spleen, brain, lung, liver, kidney, and muscle [22]. The expression levels of the NLRP1 protein were observably upregulated at 1 to 8 weeks after $\mathrm{AB}$ in WT mice. The ablation of NLRP1 alleviated pathological cardiac hypertrophy and dysfunction in response to pressure overload. Numerous factors, such as Ang-II, PE, ET-1, catecholamines, growth factors, and TNF- $\alpha$, can induce ventricular hypertrophy [23-25]. On the cellular level, the expression of the NLRP1 protein was higher after treatment with PE. Depletion of NLRP1 during treatment with PE led to the reduced size of cardiomyocyte. Cardiac hypertrophy is a common response to external stressors, including mechanical overload and neurohormonal stimulation. According to our data, ablation of NLRP1 attenuated cardiac hypertrophy induced by pressure overload and the phenylephrine-induced hypertrophic response in NRCMs; overexpression of NLRP1 promoted hypertrophy in NRCMs.

The MAPK cascade is a classical pathway involved in cardiac hypertrophy. MAPK signaling is initiated in cardiac myocytes by various stress stimuli. Once activated, downstream JNKs, p38, and ERKs phosphorylate a wide array of intracellular targets including numerous transcription factors; this results in the reprogramming of cardiac gene expression [19, 26 , 27]. NOD-like receptors (NLRs), the major constituents of the cytosolic innate immunesensing machinery, participate in multiple pathways including NF- $\mathrm{KB}$ and MAPK pathways $[28,29]$. A close relationship exists between inflammasome signaling and other signaling pathways (especially that involving TLR) [30]. The activity of inflammasome corpuscles requires a double signal stimulus. The first signal is referred to as a pre-stimulus signal, mediated by TLR or other pattern recognition receptors. The activation of MAPK and NF- 


\section{Cellular Physiology Cell Physiol Biochem 2018;48:75-86 \\ and Biochemistry Published online: July 12, $2018 \quad$\begin{tabular}{l|l} 
DOI: 2018 The Author(s). Published by S. Karger AG, Basel \\
www.karger.com/cpb
\end{tabular}}

Zong et al.: Nuclear Localization Leucine-Rich-Repeat Protein 1 Promotes Cardiac

Hypertrophy

$\kappa \mathrm{B}$ signaling pathways increases the expression of inflammatory cell-core molecules and precursors of IL-1 $\beta$ and IL-18. The second signal is called the activation signal. ATP, ROS, and other PAMPS or DAMP induce oligomerization and allosteric of NLR, recruitment of ASC, and activation of caspase-1 [31]. According to our data, the protein levels of JNK1/2, p38, and ERK1/2 phosphorylation, in response to hypertrophic stimuli, were significantly increased in NLRP1-KO mice. Our in vitro studies show that inhibition of NLRP1 expression significantly decreased the activation of JNK1/2, p38, and ERK1/2, while overexpression of NLRP1 greatly enhanced the activation of JNK1/2, p38, and ERK1/2. These findings indicate that the inhibitory effects of NLRP1 ablation on cardiac hypertrophy are mediated by MAPK signaling.

Inflammation plays an important role in the pathological progress of cardiac hypertrophy [24]. Inflammatory signaling molecules, released during cardiac remodeling, can induce hypertrophic and fibrotic responses [32]. In cardiomyocytes, major proinflammatory cytokines, such as TNF- $\alpha$, IL-1 $\beta$, and IL- 6 induce hypertrophy and promote inflammation $[33,34]$. NF- $\kappa B$ is a dimeric transcription factor that is kept inactive in the cytoplasm by inhibitory $\kappa \mathrm{B}$ proteins (i.e., I $\kappa \mathrm{Bs}$ ). The IKK/NF- $\kappa \mathrm{B}$ signaling pathway is an important regulator of inflammation. Activation of IKK/NF- $\kappa B$ is implicated in cardiac hypertrophy $[35,36]$. In adult animals, activation of IKK2 (IKK $\beta$ ) leads to inflammatory dilated cardiomyopathy and heart failure [36]. NLRs participate in NF- $\kappa \mathrm{B}$ signaling [28, 37]. According to our results, NLRP1 significantly enhanced the activation of NF- $\mathrm{BB}$ by disrupting transcriptional activity. This occurred by promoting the phosphorylation of IKK $\beta$ and I $\mathrm{KB} \alpha$, and by enhancing the expression of NF- $\kappa B$ target genes (IL-1 $\beta$, IL-10, and TNF- $\alpha$ ) in hypertrophic hearts. Our in vitro studies showed that NLRP1 depletion in NRCMs attenuated the PE-mediated hypertrophic effects, while overexpression of NLRP1 aggravated NRCM hypertrophy in response to PE stimuli. Mechanically, PE-induced activation of NF- $\mathrm{KB}$ inhibited in the NRCMs infected with Ad-shNLRP1; infection with Ad-NLRP1 increased NF- $\kappa B$ activation in NRCMs. These findings further confirm that ablation of NLRP1 protects cardiomyocytes against hypertrophy by decreasing the signaling of NF- $\kappa$ B.

Cardiac fibrosis induces pathological changes that culminate in chamber dilatation and cardiomyocyte hypertrophy. TGF- $\beta$ is upregulated in the hypertrophied and fibrotic hearts, and is regarded as one of the major profibrotic cytokines and critical mediators of cardiac fibrosis [32]. The canonical pathway of TGF- $\beta$ signaling is transduced through Smad and non-Smad pathways. The TGF- $\beta$ ligand could bind to TGF- $\beta$ R I and TGF- $\beta$ R II. TGF- $\beta$ R II phosphorylates TGF- $\beta$ R I; then, they phosphorylate and activate Smad2 and Smad3, which bind Smad 4 and translocate to the nucleus. The complex acts as a transcription factor, inducing the activation of numerous profibrotic genes [38-40]. In our study, the mRNA expression of collagen I, collagen III, and CTGF, which are responsible for cardiac fibrosis, was markedly downregulated in NLRP1-KO mice compared with the levels in WT mice in response to pressure overload. NLRP1 ablation abrogated the expression of TGF- $\beta$, TGF- $\beta R$ I, and TGF- $\beta R$ II, as well as phosphorylation of Smad2, Smad3, and Smad4 in the hypertrophied hearts, thus inhibiting fibrosis. Collectively, these data indicate that NLRP1 ablation attenuates fibrosis by blocking TGF- $\beta /$ Smad signaling.

In conclusion, this study provides the first direct evidence that NLRP1 promotes cardiac hypertrophy, inflammation, and fibrosis in response to hypertrophic stimuli by activating the MAPK, NF- $\kappa B$, and TGF- $\beta /$ Smad signaling pathways. This study helps us understand the effects of NLRP1 on cardiac hypertrophy and the related molecular mechanisms. The potential of NLRP1 as a therapeutic target will be assessed in further studies.

\section{Acknowledgements}

We thank Professor Qi-zhu Tang for his guidance with study design. This study was supported by the National Natural Science Foundation of China (Grant no. 81400178) and the Natural Science Foundation of Jiangsu Province (Grants Nos. BK20140226 and BK20160231). 


\section{Cellular Physiology Cell Physiol Biochem 2018;48:75-86 \begin{tabular}{l|l} 
and Biochemistry Published 10.1159/000491664 2018 & $\begin{array}{l}\text { () 2018 The Author(s). Published by S. Karger AG, Basel } \\
\text { www.karger.com/cpb }\end{array}$
\end{tabular}}

Zong et al.: Nuclear Localization Leucine-Rich-Repeat Protein 1 Promotes Cardiac Hypertrophy

\section{Disclosure Statement}

The authors have no conflict of interests to disclose.

\section{References}

$>1$ Frey N, Katus HA, Olson EN, Hill JA: Hypertrophy of the heart: A new therapeutic target? Circulation 2004;109:1580-1589.

-2 Levy D, Garrison RJ, Savage DD, Kannel WB, Castelli WP: Prognostic implications of echocardiographically determined left ventricular mass in the framingham heart study. N Engl J Med 1990;322:1561-1566.

-3 Kumar KR, Zhu J, Bhaskarabhatla M, Yan M, Mohan C: Enhanced expression of stem cell antigen-1 (ly-6a/e) in lymphocytes from lupus prone mice correlates with disease severity. J Autoimmun 2005;25:215-222.

4 Diwan A, Dorn GW, 2nd: Decompensation of cardiac hypertrophy: Cellular mechanisms and novel therapeutic targets. Physiology (Bethesda) 2007;22:56-64.

5 Selvetella G, Hirsch E, Notte A, Tarone G, Lembo G: Adaptive and maladaptive hypertrophic pathways: Points of convergence and divergence. Cardiovasc Res 2004;63:373-380.

6 Sundstrom J, Lind L, Arnlov J, Zethelius B, Andren B, Lithell HO: Echocardiographic and electrocardiographic diagnoses of left ventricular hypertrophy predict mortality independently of each other in a population of elderly men. Circulation 2001;103:2346-2351.

7 Topkara VK, Evans S, Zhang W, Epelman S, Staloch L, Barger PM, Mann DL: Therapeutic targeting of innate immunity in the failing heart. J Mol Cell Cardiol 2011;51:594-599.

-8 Verma SK, Krishnamurthy P, Barefield D, Singh N, Gupta R, Lambers E, Thal M, Mackie A, Hoxha E, Ramirez V, Qin G, Sadayappan S, Ghosh AK, Kishore R: Interleukin-10 treatment attenuates pressure overloadinduced hypertrophic remodeling and improves heart function via signal transducers and activators of transcription 3-dependent inhibition of nuclear factor-kappab. Circulation 2012;126:418-429.

-9 Mann DL: Inflammatory mediators and the failing heart: Past, present, and the foreseeable future. Circul res 2002;91:988-998.

$>10$ Yndestad A, Damas JK, Oie E, Ueland T, Gullestad L, Aukrust P: Role of inflammation in the progression of heart failure. Curr Cardiol Rep 2007;9:236-241.

11 Bruey JM, Bruey-Sedano N, Luciano F, Zhai D, Balpai R, Xu C, Kress CL, Bailly-Maitre B, Li X, Osterman A, Matsuzawa S, Terskikh AV, Faustin B, Reed JC: Bcl-2 and bcl-xl regulate proinflammatory caspase-1 activation by interaction with nalp1. Cell 2007;129:45-56.

12 Levandowski CB, Mailloux CM, Ferrara TM, Gowan K, Ben S, Jin Y, McFann KK, Holland PJ, Fain PR, Dinarello CA, Spritz RA: Nlrp1 haplotypes associated with vitiligo and autoimmunity increase interleukin-1beta processing via the nlrp1 inflammasome. Proc Nat Acad Sci U S A 2013;110:2952-2956.

13 Hamatani T, Falco G, Carter MG, Akutsu H, Stagg CA, Sharov AA, Dudekula DB, VanBuren V, Ko MS: Ageassociated alteration of gene expression patterns in mouse oocytes. Hum Mol Genet 2004;13:2263-2278.

-14 Hlaing T, Guo RF, Dilley KA, Loussia JM, Morrish TA, Shi MM, Vincenz C, Ward PA: Molecular cloning and characterization of defcap-l and -s, two isoforms of a novel member of the mammalian ced-4 family of apoptosis proteins. J Biol Chem 2001;276:9230-9238.

15 Fann DY, Lee SY, Manzanero S, Tang SC, Gelderblom M, Chunduri P, Bernreuther C, Glatzel M, Cheng YL, Thundyil J, Widiapradja A, Lok KZ, Foo SL, Wang YC, Li YI, Drummond GR, Basta M, Magnus T, Jo DG, Mattson MP, Sobey CG, Arumugam TV: Intravenous immunoglobulin suppresses nlrp1 and nlrp3 inflammasome-mediated neuronal death in ischemic stroke. Cell Death Dis 2013;4:e790.

-16 Kovarova M, Hesker PR, Jania L, Nguyen M, Snouwaert JN, Xiang Z, Lommatzsch SE, Huang MT, Ting JP, Koller BH: Nlrp1-dependent pyroptosis leads to acute lung injury and morbidity in mice. J Immunol 2012;189:2006-2016.

17 Bian Z, Cai J, Shen DF, Chen L, Yan L, Tang Q, Li H: Cellular repressor of e1a-stimulated genes attenuates cardiac hypertrophy and fibrosis. J Cell Mol Med 2009;13:1302-1313.

18 Yan L, Wei X, Tang QZ, Feng J, Zhang Y, Liu C, Bian ZY, Zhang LF, Chen M, Bai X, Wang AB, Fassett J, Chen Y, He YW, Yang Q Liu PP, Li H: Cardiac-specific mindin overexpression attenuates cardiac hypertrophy via blocking akt/gsk3beta and tgf-beta1-smad signalling. Cardiovas Res 2011;92:85-94. 


\section{Cellular Physiology Cell Physiol Biochem 2018:48:75-86 \begin{tabular}{l|l} 
and Biochemistry Published 10.1159/000491664 2018 & $\begin{array}{l}\text { () 2018 The Author(s). Published by S. Karger AG, Basel } \\
\text { www.karger.com/cpb }\end{array}$
\end{tabular}}

Zong et al.: Nuclear Localization Leucine-Rich-Repeat Protein 1 Promotes Cardiac Hypertrophy

19 Zong J, Salim M, Zhou H, Bian ZY, Dai J, Yuan Y, Deng W, Zhang JY, Zhang R, Wu QQ Tang QZ: Nod2 deletion promotes cardiac hypertrophy and fibrosis induced by pressure overload. Lab Inves 2013;93:1128-1136.

-20 Ting JP, Lovering RC, Alnemri ES, Bertin J, Boss JM, Davis BK, Flavell RA, Girardin SE, Godzik A, Harton JA, Hoffman HM, Hugot JP, Inohara N, Mackenzie A, Maltais LJ, Nunez G, Ogura Y, Otten LA, Philpott D, Reed JC, Reith W, Schreiber S, Steimle V, Ward PA: The nlr gene family: A standard nomenclature. Immunity 2008;28:285-287.

21 Boyden ED, Dietrich WF: Nalp1b controls mouse macrophage susceptibility to anthrax lethal toxin. Nat Genet 2006;38:240-244.

-22 Lech M, Avila-Ferrufino A, Skuginna V, Susanti HE, Anders HJ: Quantitative expression of rig-like helicase, nod-like receptor and inflammasome-related mrnas in humans and mice. Int Immunol 2010;22:717-728.

23 Zhang X, Xia J, Qian D, Wang Y, Lin Y, Huang X, Tan J: An adenosine a1 agonist 2-chloro-n6 cyclopentyladenosine inhibits the angiotensin ii-induced cardiomyocyte hypertrophy through the calcineurin pathway. Cardiology 2014;129:153-162.

24 Guan XH, Hong X, Zhao N, Liu XH, Xiao YF, Chen TT, Deng LB, Wang XL, Wang JB, Ji GJ, Fu M, Deng KY, Xin HB: Cd38 promotes angiotensin ii-induced cardiac hypertrophy. J cell mol med 2017;21:1492-1502.

25 Wang PX, Li ZM, Cai SD, Li JY, He P, Huang Y, Feng GS, Luo HB, Chen SR, Liu PQ: C33(s), a novel pde9a inhibitor, protects against rat cardiac hypertrophy through upregulating cgmp signaling. Acta Pharmacol Sin 2017;38:1257-1268.

-26 Rose BA, Force T, Wang Y: Mitogen-activated protein kinase signaling in the heart: Angels versus demons in a heart-breaking tale. Physiol Rev 2010;90:1507-1546.

27 Huang H, Tang QZ, Wang AB, Chen M, Yan L, Liu C, Jiang H, Yang Q, Bian ZY, Bai X, Zhu LH, Wang L, Li H: Tumor suppressor a20 protects against cardiac hypertrophy and fibrosis by blocking transforming growth factor-beta-activated kinase 1-dependent signaling. Hypertension 2010;56:232-239.

28 Lupfer C, Kanneganti TD: The expanding role of nlrs in antiviral immunity. Immunol Rev 2013;255:13-24.

-29 Liu R, Truax AD, Chen L, Hu P, Li Z, Chen J, Song C, Chen L, Ting JP: Expression profile of innate immune receptors, nlrs and aim2, in human colorectal cancer: Correlation with cancer stages and inflammasome components. Oncotarget 2015;6:33456-33469.

-30 Chi W, Li F, Chen H, Wang Y, Zhu Y, Yang X, Zhu J, Wu F, Ouyang H, Ge J, Weinreb RN, Zhang K, Zhuo Y: Caspase-8 promotes nlrp1/nlrp3 inflammasome activation and il-1beta production in acute glaucoma. Proc Nat Acad Sci U S A 2014;111:11181-11186.

31 Guo H, Callaway JB, Ting JP: Inflammasomes: Mechanism of action, role in disease, and therapeutics. Nature Med 2015;21:677-687.

-32 Frieler RA, Mortensen RM: Immune cell and other noncardiomyocyte regulation of cardiac hypertrophy and remodeling. Circulation 2015;131:1019-1030.

33 Siwik DA, Chang DL, Colucci WS: Interleukin-1beta and tumor necrosis factor-alpha decrease collagen synthesis and increase matrix metalloproteinase activity in cardiac fibroblasts in vitro. Circul Res 2000;86:1259-1265.

-34 Yokoyama T, Nakano M, Bednarczyk JL, McIntyre BW, Entman M, Mann DL: Tumor necrosis factor-alpha provokes a hypertrophic growth response in adult cardiac myocytes. Circulation 1997;95:1247-1252.

35 Van der Heiden K, Cuhlmann S, Luong le A, Zakkar M, Evans PC: Role of nuclear factor kappab in cardiovascular health and disease. Clinical Sci 2010;118:593-605.

-36 Maier HJ, Schips TG, Wietelmann A, Kruger M, Brunner C, Sauter M, Klingel K, Bottger T, Braun T, Wirth T: Cardiomyocyte-specific ikappab kinase (ikk)/nf-kappab activation induces reversible inflammatory cardiomyopathy and heart failure. Proc Nat Acad Sci USA 2012;109:11794-11799.

-37 Hu W, Zhang Y, Wu W, Yin Y, Huang D, Wang Y, Li W, Li W: Chronic glucocorticoids exposure enhances neurodegeneration in the frontal cortex and hippocampus via nlrp-1 inflammasome activation in male mice. Brain behav Immun 2016;52:58-70.

38 Bujak M, Frangogiannis NG: The role of tgf-beta signaling in myocardial infarction and cardiac remodeling. Cardiovascul Res 2007;74:184-195.

-39 Bujak M, Ren G, Kweon HJ, Dobaczewski M, Reddy A, Taffet G, Wang XF, Frangogiannis NG: Essential role of smad3 in infarct healing and in the pathogenesis of cardiac remodeling. Circulation 2007;116:2127-2138.

40 Travers JG, Kamal FA, Robbins J, Yutzey KE, Blaxall BC: Cardiac fibrosis: The fibroblast awakens. Circul Res 2016;118:1021-1040. 